\title{
Suction against resistance: a new breathing technique to significantly improve the blood flow ratio of the superior and inferior vena cava
}

\author{
Andreas Gutzeit • Justus E. Roos • Klaus Hergan • \\ Constantin von Weymarn • Stephan Wälti • \\ Carolin Reischauer • Johannes M. Froehlich
}

Received: 9 January 2014 / Revised: 23 June 2014 / Accepted: 8 July 2014 / Published online: 9 August 2014

(C) European Society of Radiology 2014

\begin{abstract}
Objectives Optimal contrast within the pulmonary artery is achieved by the maximum amount of contrast-enhanced blood flowing through the superior vena cava (SVC), while minimum amounts of non-contrasted blood should originate from the inferior vena cava (IVC). This study aims to clarify whether "suction against resistance" might optimise this ratio.

Methods Phase-contrast pulse sequences on a 1.5T MRI magnet were used for flow quantification (mean flow $(\mathrm{mL} / \mathrm{s})$, stroke volume (Vol) in the SVC and IVC in volunteers. Different breathing manoeuvers were analysed repeatedly: free breathing; inspiration; expiration; suction against resistance, and Valsalva. To standardise breathing commands, volunteers performed suction and Valsalva manoeuvers with an MR-compatible manometer.

Results Suction against resistance was associated with a significant drop of the IVC/SVC flow quotient (1.63 [range 1.32.0] $p<0.05$ at $-10 \mathrm{mmHg}$ and 1.48 [1.1-1.9] $p<0.01$ at -
\end{abstract}

A. Gutzeit $(\bowtie) \cdot$ C. von Weymarn · C. Reischauer · J. M. Froehlich Institute of Radiology and Nuclear Medicine, Clinical Research Unit of St. Anna Hospital Luzern, St. Anna Strasse 32, 6006 Luzern, Switzerland

e-mail: Andreas.Gutzeit@hirslanden.ch

A. Gutzeit $\cdot$ K. Hergan

Department of Radiology, Paracelsus Medical University Salzburg, Salzburg, Austria

A. Gutzeit $\cdot$ C. von Weymarn $\cdot$ S. Wälti $\cdot$ C. Reischauer •

J. M. Froehlich

Department of Radiology, Cantonal Hospital, Winterthur,

Switzerland

J. E. Roos

Department of Radiology, Duke University, Durham, NC, USA
$20 \mathrm{mmHg}$ ) corresponding to increased blood flow from SVC and diminished flow originating from the IVC. The remaining breathing commands (free breathing 2.2; inspiration 2.4; expiration 2.4; Valsalva $10 \mathrm{mmHg} 2.3$; Valsalva $20 \mathrm{mmHg}$ 2.6; and Valsalva $30 \mathrm{mmHg} 2.2$ ) showed no differences $(p>0.05)$.

Conclusions Suction against resistance caused a significant drop in the IVC/SVC quotient. Theoretically, this breathing manoeuver might significantly improve the enhancement characteristics of CT angiography.

Key Points

- Suction provokes reduction in blood flow in the inferior vena cava.

- Ratio between the inferior and superior vena cava blood flow diminished during suction.

- Manometer used during breathing standardises MR phasecontrast blood flow measurements.

Keywords Magnetic resonance imaging · Phase-contrast . Breathing $\cdot$ Suction $\cdot$ Inferior vena cava $\cdot$ Superior vena cava

\section{Introduction}

Computer tomography (CT) angiography is widely accepted as the gold standard for the examination of patients with suspected pulmonary embolism (PE) [1]. The advantages of CT are obvious: it is widely available, the method is fast, and it is highly sensitive for the diagnosis of PE. In fact, in clinical studies, the sensitivity of CT angiography is reported to range between $53 \%$ and $100 \%$, while its specificity ranges between $83 \%$ and $100 \%$ for diagnosing PE [2, 3]. Indeed, high contrast within the pulmonary artery is essential to diagnose the presence of PE with CT angiography. Moreover, insufficient contrast enhancement in the pulmonary artery may 
mimic a false-positive embolus [4]. Pulmonary circulatory status is highly dependable on cardiac output and intrathoracic pressure conditions, which are variable during different breathing manoeuvers. Discussion of different factors influencing the quality of pulmonary CT angiography is a subject of ongoing debate, and there are partially contradictory research results [5-7]. From the physiological point of view, the prerequisites for optimal contrast within the pulmonary artery are quite clear. While administering the contrast medium via venous access over the upper extremity (most popular venous access is the back of the hand or antecubital vein), the maximum amount of contrastenhanced blood should flow through the superior vena cava (SVC) into the right atrium, and a minimum volume of noncontrasted blood should reach the heart from the inferior vena cava (IVC). Evidently, the proportion of non-contrasted blood of the IVC in relation to the SVC influences the dilution of contrast medium in the right atrium, the ventricle, and finally in the pulmonary artery (PA), also called the "transient interruption of contrast bolus" [7].

Summarising numerous publications, expiration followed by the apnoea phase seems to be superior to other respiratory manoeuvers. Various pulmonary CT studies have been conducted confirming that contrast in PA after expiration with apnoea is improved compared to inspiration with apnoea [5-7]. However, the discussion is controversial and has not yet been conclusively resolved [8-10].

In order to evaluate the influence of standardised breathing manoeuvers, including the newly introduced suction against resistance scheme here, we planned to quantify the blood flow rates in the SVC and IVC. For this purpose, we used an MR quantification method without radiation to further investigate the physiological background.

Finally the goal of the study was to determine the optimal breathing manoeuver yielding the lowest IVC/SVC blood flow ratio, thus, potentially allowing improvement of contrast-enhanced exams in the future.

\section{Materials and methods}

\section{Volunteers}

This prospective study was conducted from November 2012 to February 2013, in accordance with the Declaration of Helsinki, after obtaining approval from the institutional review board. Written informed consent was obtained from all volunteers.

Overall, 26 subjects were included in this study (12 males, 14 females; mean age 27 years, age range 25-55 years), while four had to be excluded during the study due to claustrophobia or inability to comply with the study protocol. Thus, the perprotocol analysis consists of 22 subjects. Further on, no volunteer had a history of pulmonary or cardiovascular disease. The mean body mass index (BMI) was 22.3 (range, 20 to 27.8). The volunteers were healthy staff members from our hospital.

\section{MRI examination}

Subjects were imaged in the supine position on a 1.5 Tesla MRI unit (Achieva 1.5 T, Philips Healthcare, Best, the Netherlands). We used an 8-channel torso coil (Philips Healthcare) covering the entire chest allowing the regular acquisition of two sets of hearttriggered dynamic $2 \mathrm{D}$ phase contrast $(\mathrm{PC})$ images $(\mathrm{TR}=5.3 \mathrm{~ms}$ and $\mathrm{TE}=3 \mathrm{~ms}$; slice thickness $8 \mathrm{~mm}$, flip angle $=15^{\circ}$, velocity encoding $=100 \mathrm{~ms}$; in-plane pixel size $=1.9 \times 2.5 \mathrm{~mm}^{2}$ ). Using ECG data, measurements were retrospectively assigned to the corresponding heart cycle phase. Volunteers underwent a longer training session, explaining to them in detail the various breathing manoeuvers. Measurements in the SVC and the IVC were performed in two separate examinations. Blood flow in the SVC was measured between the azygos confluence and the right atrium, while blood flow in the IVC was measured between the portal vein and the confluence to the right atrium (Fig. 1). The sequence used required at least $19 \mathrm{~s}$ of breath-holding and the measurement time depended on the volunteers' cardiac cycle.

MR compatible manometer to standardise suction and Valsalva manoeuver

To perform standardised and reproducible allocations of Valsalva manoeuvers and suction, we developed an MR-compatible manometer consisting of a non-magnetic pressure measurement device and a plastic tube. The manometer itself was, therefore, largely composed of precious metals, preventing artefacts or image deflections. The device could be used inside the scanner to perform pressure controlled Valsalva manoeuvers at $+10,+20$, or $+30 \mathrm{mmHg}$, respectively. Furthermore, we also adopted a negative manometer, which permitted suction modulation at 10 and $-20 \mathrm{mmHg}$ (Fig. 2). When using the manometer, the volunteer was either asked to perform a Valsalva manoeuver while blowing continuously into the mouthpiece (overpressure), or otherwise to aspirate continuously in a wellcontrolled manner to reach underpressure (suction against resistance). The manometer allows continuous monitoring and precise standardisation of the pressure conditions during the breathing manoeuvers.

\section{Breathing manoeuvers}

The investigation protocol included the following measurements in the following order: $\boldsymbol{A}$ : Free breathing. $\boldsymbol{B}$. Normal inspiration followed by a breath hold through the open mouth. $\boldsymbol{C}$. Normal expiration followed by a breath hold through the open mouth. $\boldsymbol{D}$. Valsalva manoeuver through the tube keeping an overpressure of $+10 \mathrm{mmHg}$. $\boldsymbol{E}$. Valsalva $+20 \mathrm{mmHg}, \boldsymbol{F}$. Valsalva $+30 \mathrm{mmHg}$. G. "Suction against resistance" 
Fig. 1 Blood flow in the SVC was measured between the azygos confluence and the right atrium $(\mathbf{a}+\mathbf{b})$ on the identical axial plane using the phasecontrast acquisitions (b, d). Accordingly, blood flow in the IVC was measured between the portal vein and the confluence to the right atrium $(\mathbf{c}+\mathbf{d})$

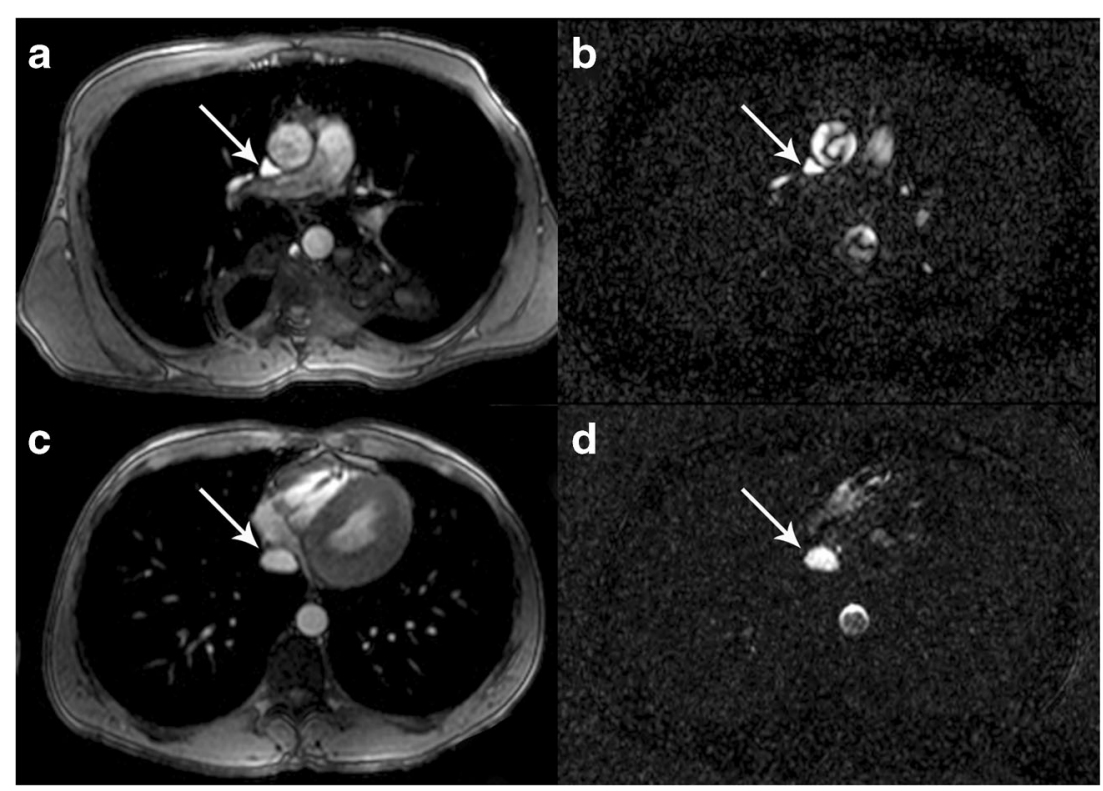

corresponding to aspiration through the tube maintaining a constant negative pressure of $-10 \mathrm{mmHg}, \boldsymbol{H}$ "Suction against resistance" at $-20 \mathrm{mmHg}$ (Fig. 3).

To prove reproducibility, all individual respiration manoeuvers and their flow quantifications were repeated three times by each volunteer, respectively.

\section{Quantification of blood flow in the SVC and IVC}

MR data were transferred to a workstation (EWS, Philips Healthcare) with dedicated cardiac software allowing PC flow measurements. Regions of interest were marked by a welltrained radiologist (AG; 12 years of experience) on the modulus image covering either the SVC or IVC and automatically copied to the respective PC image (Fig. 1). Quantitative data originating

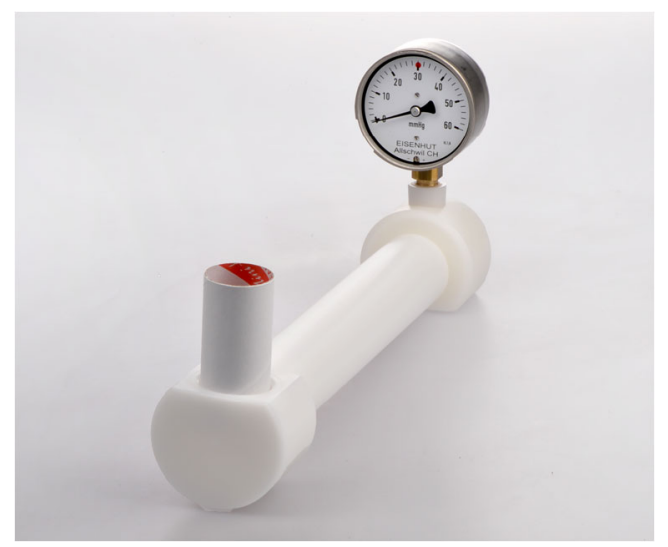

Fig. 2 Figure 2 shows the MR-compatible manometer including the mouth piece, which the volunteer had to hold in his hand during the investigation. Thus, we were able to perform investigations under standardised conditions with target positive pressures of either $+10,+20$ or $+30 \mathrm{mmHg}$, respectively, or at negative pressures of -10 and $-20 \mathrm{mmHg}$ (suction against resistance), respectively from the PC images were evaluated and yielded flow $(\mathrm{mL} / \mathrm{s})$ and absolute stroke volume $(\mathrm{mL})$ for each respiratory manoeuver.

Statistical analysis

The means of the three repetitive measurements (for each measurement A, B, C, D, E, F, G and $\mathrm{H}$ ) were calculated. The number of observations $(n)$, minimum, maximum, mean, and standard deviation (SD) are given for each breathing manoeuver. Means and the $95 \%$ confidence interval $(95 \% \mathrm{CI})$ were calculated for the various resulting IVC/SVC ratios. A repeated measurement of ANOVA with Dunnett's correction for multiple testing was performed to compare all breathing methods with free breathing (A) as a standard of reference. To test repeatability between the three measurements of each breathing manoeuver, the intraclass correlation coefficient (ICC) was calculated with a one-way random model. Single measurements are shown.

SPSS version 20 and GraphPad Prism Version 5 (IBM, SPSS statistics) were used.

\section{Results}

The quantitative analyses of the IVC and SVC stroke volumes $(\mathrm{VOL})$ and flow data $(\mathrm{mL} / \mathrm{s})$ are summarised in Tables 1 and 2 , respectively.

Overall, we were faced with four failed measurements in the SVC during the Valsalva manoeuver at $+30 \mathrm{mmHg}$ and two failed measurements in the SVC after suction at $-20 \mathrm{mmHg}$ (Tables 1 and 2). For the IVC we had two failed measurements at $+30 \mathrm{mmHg}$ and four failed measurements after suction at $10 \mathrm{mmHg}$. The missing data resulted from the fact that the 
Fig. 3 The complete experimental set-up including physiology is shown here. During free breathing, the diaphragm rises and falls. During a Valsalva manoeuver, the diaphragm rises markedly with high intrathoracic pressure. During a suction against resistance manoeuver, the diaphragm moves downwards and high underpressure in the thorax results. These intrathoracic pressure differences create markedly different flow conditions in the SVC and the IVC. To explain the breathing manoeuver further, we have added the two most extreme manometer positions. Free breathing, inspiration with apnoea and expiration with apnoea were all performed without pressure control of such a breathing device

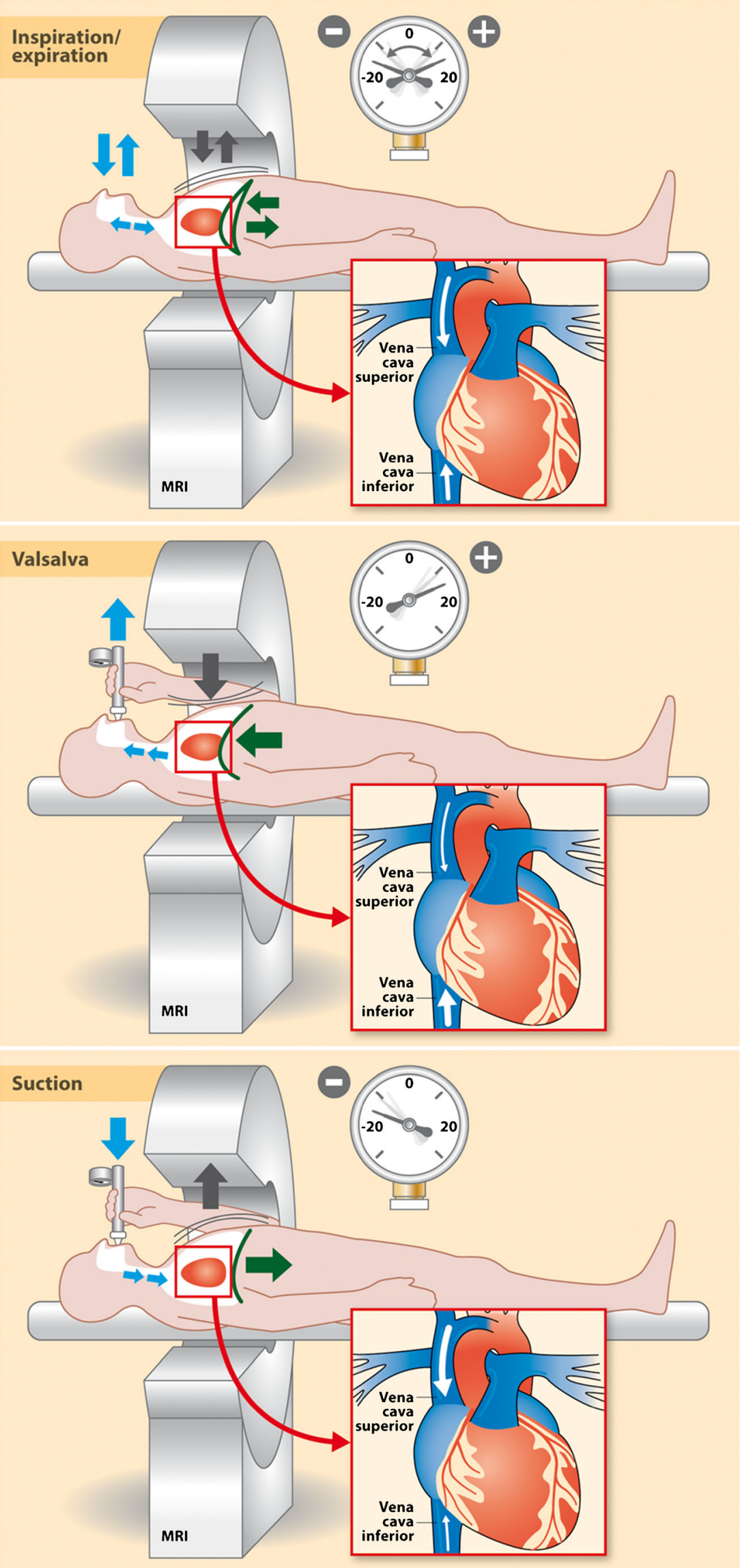


Table 1 Minimum, maximum, mean and standard deviations (SD) for stroke volumes and flow in the SVC measured with different breathing methods ( $N=$ number of individuals)

\begin{tabular}{lllllll}
\hline & Breathing method & N & Minimum & Maximum & Mean & SD \\
\hline Stroke (vol) & Free breathing & 22 & 18.87 & 42.07 & 28.37 & 5.65 \\
& Inspiration end & 22 & 17.13 & 37.07 & 26.94 & 6.34 \\
& Expiration end & 22 & 17.83 & 39.57 & 26.89 & 5.87 \\
& $10 \mathrm{~mm} \mathrm{Hg}$ & 22 & 17.97 & 36.80 & 26.85 & 5.32 \\
& $20 \mathrm{~mm} \mathrm{Hg}$ & 22 & 11.53 & 36.07 & 21.40 & 5.86 \\
& $30 \mathrm{~mm} \mathrm{Hg}$ & 18 & 13.27 & 37.77 & 22.07 & 6.89 \\
& $-10 \mathrm{~mm} \mathrm{Hg}$ & 22 & 19.50 & 43.03 & 30.75 & 6.30 \\
& $-20 \mathrm{~mm} \mathrm{Hg}$ & 20 & 18.20 & 41.50 & 29.41 & 6.49 \\
& Free breathing & 22 & 19.00 & 41.97 & 31.54 & 6.60 \\
& Inspiration end & 22 & 16.63 & 49.47 & 30.08 & 9.23 \\
& Expiration end & 22 & 16.73 & 47.97 & 30.20 & 7.50 \\
& $10 \mathrm{~mm} \mathrm{Hg}$ & 22 & 20.37 & 48.40 & 30.28 & 7.16 \\
& $20 \mathrm{~mm} \mathrm{Hg}$ & 22 & 15.27 & 43.63 & 26.07 & 6.99 \\
& $30 \mathrm{~mm} \mathrm{Hg}$ & 18 & 17.37 & 45.70 & 26.84 & 7.07 \\
& $-10 \mathrm{~mm} \mathrm{Hg}$ & 22 & 22.43 & 45.07 & 33.89 & 6.82 \\
& $-20 \mathrm{~mm} \mathrm{Hg}$ & 20 & 18.53 & 44.40 & 33.76 & 7.40 \\
\hline
\end{tabular}

volunteers were physically unable to perform these challenging manoeuvers due to ECG- related prolongment of measurement. These incomplete measurements were secondarily excluded from the analyses.

To illustrate in more detail the proportion of IVC and SVC blood flow under different respiratory manoeuvers, we analysed the volume and flow ratios (IVC/SVC) (Table 3, Fig. 4) in detail. The data clearly demonstrate that the majority of respiratory manoeuvers reveal minor blood flow changes compared to the respective reference during free breathing. The stroke volume and flow ratios during free breathing, inspiration and apnoea, expiration and apnoea, and after Valsalva manoeuvers at $+10,+$
20 and $+30 \mathrm{mmHg}$ yielded no statistically significant differences between the single manoeuvers $(p>0.05)$. However, the IVC/ SVC stroke volume and flow ratios were markedly reduced during the standardised suction manoeuvers at $-10 \mathrm{mmHg}(p$ value $<0.05)$, and even more relevant during suction at $20 \mathrm{mmHg}(p<0.01)$ (Table 4). Thus, the IVC/SVC ratios drop significantly during suction against resistance with a negative pressure of -10 or $-20 \mathrm{mmHg}$.

The three repetitive measurements of every respiratory manoeuver revealed robust values for all respiratory manoeuvers with high intraclass correlation (ICC) ranging from 0.8 to 1.0 (Table 5).
Table 2 Minimum, maximum, mean and SD for stroke and flow in the IVC measured with different breathing methods

\begin{tabular}{lllllll}
\hline & Breathing method & N & Minimum & Maximum & Mean & SD \\
\hline \multirow{2}{*}{ Stroke (vol) } & Free breathing & 22 & 38.13 & 86.60 & 60.93 & 11.33 \\
& Inspiration end & 22 & 39.97 & 80.53 & 63.21 & 11.47 \\
& Expiration end & 22 & 42.07 & 78.77 & 62.87 & 10.63 \\
& $10 \mathrm{~mm} \mathrm{Hg}$ & 22 & 27.70 & 85.53 & 62.15 & 12.38 \\
& $20 \mathrm{~mm} \mathrm{Hg}$ & 22 & 21.93 & 71.23 & 53.46 & 11.85 \\
& $30 \mathrm{~mm} \mathrm{Hg}$ & 18 & 33.83 & 69.20 & 49.51 & 11.42 \\
& $-10 \mathrm{~mm} \mathrm{Hg}$ & 22 & 2.70 & 69.20 & 49.57 & 18.87 \\
& $-20 \mathrm{~mm} \mathrm{Hg}$ & 20 & 4.70 & 70.67 & 41.99 & 22.20 \\
& Free breathing & 22 & 48.73 & 93.83 & 67.75 & 13.73 \\
& Inspiration end & 22 & 48.23 & 101.60 & 69.95 & 16.62 \\
& Expiration end & 22 & 53.50 & 89.40 & 70.23 & 12.38 \\
& $10 \mathrm{~mm} \mathrm{Hg}$ & 22 & 44.30 & 98.17 & 69.05 & 13.99 \\
& $20 \mathrm{~mm} \mathrm{Hg}$ & 22 & 43.33 & 91.47 & 64.93 & 14.33 \\
& $30 \mathrm{~mm} \mathrm{Hg}$ & 20 & 5.87 & 90.17 & 47.11 & 24.57 \\
& $-10 \mathrm{~mm} \mathrm{Hg}$ & 18 & 25.10 & 81.50 & 58.19 & 13.71 \\
& $-20 \mathrm{~mm} \mathrm{Hg}$ & 22 & 2.73 & 85.07 & 52.38 & 18.75 \\
\hline
\end{tabular}


Table 3 IVC/SVC ratios. Data show the mean and $95 \%$ CI. It could be shown that suction against resistance $(-10$ and $-20 \mathrm{mmHg})$ strongly reduces the ratios, corresponding to a significant $(p<0.01$ for $-20 \mathrm{mmHg}$; $p<0.05$ for $-10 \mathrm{mmHg}$ ) blood flow rise in the SVC accompanied by reduced blood flow from the IVC

\begin{tabular}{lllll}
\hline & Ratio IVC/SVC & $\mathrm{N}$ & Mean & $95 \% \mathrm{CI}$ \\
\hline Stroke (vol) & Free breathing & 22 & 2.20 & $1.99-2.42$ \\
& Inspiration end & 22 & 2.43 & $2.18-2.67$ \\
& Expiration end & 22 & 2.40 & $2.19-2.61$ \\
& $10 \mathrm{~mm} \mathrm{Hg}$ & 22 & 2.36 & $2.13-2.59$ \\
& $20 \mathrm{~mm} \mathrm{Hg}$ & 22 & 2.57 & $2.32-2.83$ \\
& $30 \mathrm{~mm} \mathrm{Hg}$ & 18 & 2.32 & $2.11-2.53$ \\
& $-10 \mathrm{~mm} \mathrm{Hg}$ & 22 & $\mathbf{1 . 6 7}$ & $1.34-1.99$ \\
& $-20 \mathrm{~mm} \mathrm{Hg}$ & 20 & $\mathbf{1 . 4 8}$ & $1.08-1.88$ \\
Flow (mL/s) & Free breathing & 22 & 2.20 & $1.99-2.42$ \\
& Inspiration end & 22 & 2.43 & $2.18-2.67$ \\
& Expiration end & 22 & 2.40 & $2.20-2.61$ \\
& $10 \mathrm{~mm} \mathrm{Hg}$ & 22 & 2.35 & $2.12-2.59$ \\
& $20 \mathrm{~mm} \mathrm{Hg}$ & 22 & 2.57 & $2.32-2.83$ \\
& $30 \mathrm{~mm} \mathrm{Hg}$ & 18 & 2.25 & $1.97-2.52$ \\
& $-10 \mathrm{~mm} \mathrm{Hg}$ & 22 & $\mathbf{1 . 6 3}$ & $1.30-1.96$ \\
& $-20 \mathrm{~mm} \mathrm{Hg}$ & 20 & $\mathbf{1 . 4 8}$ & $1.08-1.88$ \\
\hline
\end{tabular}

\section{Discussion}

In this study, 22 volunteers were examined during various standardised breathing manoeuvers using flow-sensitive MR phase contrast techniques in the SVC and IVC. In order to guarantee standardised and reproducible breathing, an MRcompatible closed manometer system was used for monitoring of the respiratory pressure during the entire manoeuver.

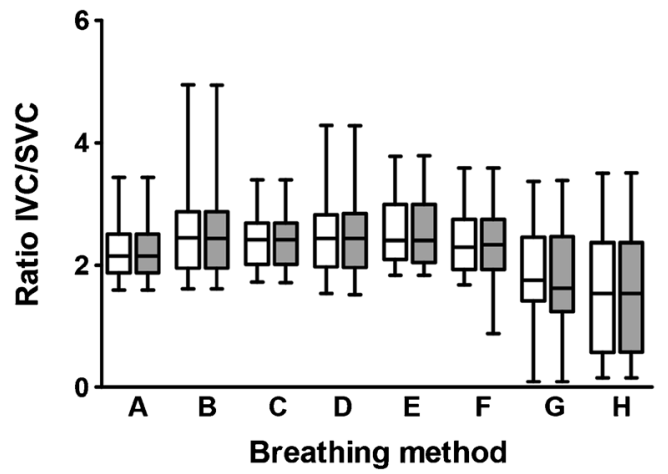

Fig. 4 IVC/SVC ratios for stroke volumes (white boxes) and flow (grey boxes) for free breathing (A), end of inspiration position with breath hold (B), end of expiration position with breath hold (C), Valsalva manoeuver at $+10 \mathrm{mmHg}$ (D), Valsalva manoeuver at $+20 \mathrm{mmHg}$ (E), Valsalva manoeuver at $+30 \mathrm{mmHg}(\mathrm{F})$, suction manoeuver at $-10 \mathrm{mmHg}(\mathrm{G})$, and similar suction manoeuver at $-20 \mathrm{mmHg}(\mathrm{H})$. Boxes show the median and the 25th and 75th quartiles; whiskers show minimum and maximum values. The optimal ratio is achieved in the suction mode with thoracic underpressure, but standard deviations are slightly higher, demonstrating more unstable conditions
Thus, it could be shown that "suction against resistance" yields highly favourable SVC and IVC blood flow and volume ratios with markedly increased SVC blood flow accompanied by strongly reduced blood flow in the IVC. As a result, the ratio of IVC/SVC decreased significantly compared to all the other respiratory manoeuvers $(p<0.001)$. The other respiratory manoeuvres such as Valsalva, apnoea after end of expiration, apnoea after end of inspiration, and free breathing all yielded similar ratios, but were all markedly inferior compared to suction against resistance. The measurements were repeated several times and compared with one another. The ICC score was predominantly between 0.8 and 1.0 , which demonstrated very robust reproducibility and repeatability of our measurement method.

According to several previously published CT angiography studies, the impact of different breathing manoeuvers on pulmonary arterial enhancement was discussed controversially [5-10]. However, it should be emphasized that all of these studies were CT investigations. The main shortcoming of these CT studies was the fact that no dynamic and real-time measurements relative to different breathing manoeuvers were possible in contrast to MRI investigations. The majority of radiological studies presume that apnoea after the end of inspiration exerts rather a negative impact on contrast enhancement of pulmonary arteries [4-7]. The different authors explain this phenomenon by the fact that during inspiration the diaphragm moves in the caudal direction, thus, exerting pressure on abdominal organs and passively to the inferior vena cava. This abdominal pressure might indirectly increase non-enhanced blood flow from the IVC towards the heart resulting in increased dilution in the right atrium and transient interruption of contrast bolus [7].

In contrast to previous studies, our MR study shows that suction against resistance (which is similar to the Müller manoeuver) presents the highest reduction of IVC/SVC ratio. This seems to be of great practical relevance especially when implementing this knowledge for optimisation of contrastenhanced CT angiography in further prospective investigations. We have to emphasise that the resulting high flow from the SVC should consequently improve CT attenuation of the pulmonary artery because suction against resistance significantly reduces dilution from non-enhanced blood originating from the IVC.

Interestingly, the other breathing manoeuvers did not significantly change the IVC/SVC ratios. Similar to our study, the various effects of breathing on the SVC and the IVC were recently also examined in a further MR study by Kuzo et al. [11]. In contrast to our study, the authors concluded that mild continuous inspiration has rather an increasingly negative effect on the IVC blood flow [11]. This earlier result apparently confirms the general observation of radiological CT studies, which described contrast enhancement of pulmonary arteries on CT during inspiration as well reduced [4-7]. But 
Table 4 Dunnett's multiple comparison test for the IVC/SVC ratios in comparison to free breathing as a standard of reference. A total of 17 volunteers with valid data for all eight breathing methods were included. The two suction modes differed statistically significantly (flow and stroke volume) from free breathing $(p<0.05$ in the case of suction with $-10 \mathrm{mmHg}$ and $\mathrm{p}<0.01$ with suction of $-20 \mathrm{mmHg}$ ), while all other manoeuvers did not show a significant change

\begin{tabular}{|c|c|c|c|c|}
\hline & Free breathing vs & Mean difference & $95 \% \mathrm{CI}$ & p-value \\
\hline \multirow[t]{7}{*}{ Stroke } & Inspiration end & -0.3765 & $-0.7629-0.009973$ & $>0.05$ \\
\hline & Expiration end & -0.1235 & $-0.5100-0.2629$ & $>0.05$ \\
\hline & $10 \mathrm{~mm} \mathrm{Hg}$ & -0.2512 & $-0.6376-0.1353$ & $>0.05$ \\
\hline & $20 \mathrm{~mm} \mathrm{Hg}$ & -0.3159 & $-0.7023-0.07056$ & $>0.05$ \\
\hline & $30 \mathrm{~mm} \mathrm{Hg}$ & -0.1376 & $-0.5241-0.2488$ & $>0.05$ \\
\hline & $-10 \mathrm{~mm} \mathrm{Hg}$ & 0.3888 & $0.002380-0.7753$ & $<0.05$ \\
\hline & $-20 \mathrm{~mm} \mathrm{Hg}$ & 0.5676 & $0.1812-0.9541$ & $<0.01$ \\
\hline \multirow[t]{7}{*}{ Flow } & Inspiration end & -0.3706 & $-0.7625-0.02133$ & $>0.05$ \\
\hline & Expiration end & -0.1171 & $-0.5090-0.2749$ & $>0.05$ \\
\hline & $10 \mathrm{~mm} \mathrm{Hg}$ & -0.2400 & $-0.6319-0.1519$ & $>0.05$ \\
\hline & $20 \mathrm{~mm} \mathrm{Hg}$ & -0.3065 & $-0.6984-0.08545$ & $>0.05$ \\
\hline & $30 \mathrm{~mm} \mathrm{Hg}$ & -0.1082 & $-0.5002-0.2837$ & $>0.05$ \\
\hline & $-10 \mathrm{~mm} \mathrm{Hg}$ & 0.4388 & $0.04691-0.8307$ & $<0.05$ \\
\hline & $-20 \mathrm{~mm} \mathrm{Hg}$ & 0.5718 & $0.1798-0.9637$ & $<0.01$ \\
\hline
\end{tabular}

how can one explain this rather contradictory result described by Kuzo et al., in comparison to ours?

To better understand this, one has to take a very close look at the precise definition of breathing manoeuvers assessed in all previously published studies. Kuzo's so-called continuous inspiration method differs greatly from our suction against resistance negative pressure manoeuver, as we ask our volunteers to exert continuous suction against resistance guaranteed by a closed manometer tube. In contrast, Kuzo's continuous

Table 5 Reproducibility of the three different measurements of each individual manoeuver show high intraclass correlation (ICC) scores, demonstrating that the study design and measurements are reliable

\begin{tabular}{|c|c|c|c|c|}
\hline & \multirow{2}{*}{$\begin{array}{l}\text { Breathing } \\
\text { method }\end{array}$} & \multirow[t]{2}{*}{$\mathrm{N}$} & \multicolumn{2}{|l|}{ ICC (95 \% CI) } \\
\hline & & & Stroke & Flow \\
\hline \multirow[t]{8}{*}{ SVC } & Free breathing & 22 & $0.836(0.703-0.922)$ & $0.831(0.694-0.919)$ \\
\hline & Inspiration end & 22 & $0.880(0.776-0.944)$ & $0.913(0.834-0.960)$ \\
\hline & Expiration end & 22 & $0.913(0.834-0.960)$ & $0.906(0.822-0.956)$ \\
\hline & $10 \mathrm{~mm} \mathrm{Hg}$ & 22 & $0.872(0.762-0.940)$ & $0.893(0.799-0.950)$ \\
\hline & $20 \mathrm{~mm} \mathrm{Hg}$ & 22 & $0.921(0.848-0.963)$ & $0.924(0.854-0.965)$ \\
\hline & $30 \mathrm{~mm} \mathrm{Hg}$ & 16 & $0.791(0.597-0.913)$ & $0.744(0.521-0.891)$ \\
\hline & $-10 \mathrm{~mm} \mathrm{Hg}$ & 22 & $0.868(0.756-0.938)$ & $0.816(0.670-0.911)$ \\
\hline & $-20 \mathrm{~mm} \mathrm{Hg}$ & 20 & $0.811(0.654-0.913)$ & $0.817(0.663-0.916)$ \\
\hline \multirow[t]{8}{*}{ IVC } & Free breathing & 22 & $0.836(0.703-0.922)$ & $0.831(0.694-0.919)$ \\
\hline & Inspiration end & 22 & $0.880(0.776-0.944)$ & $0.913(0.834-0.960)$ \\
\hline & Expiration end & 22 & $0.913(0.834-0.960)$ & $0.906(0.822-0.956)$ \\
\hline & $10 \mathrm{~mm} \mathrm{Hg}$ & 22 & $0.872(0.762-0.940)$ & $0.893(0.799-0.950)$ \\
\hline & $20 \mathrm{~mm} \mathrm{Hg}$ & 22 & $0.921(0.848-0.963)$ & $0.924(0.854-0.965)$ \\
\hline & $30 \mathrm{~mm} \mathrm{Hg}$ & 16 & $0.791(0.597-0.913)$ & $0.744(0.521-0.891)$ \\
\hline & $-10 \mathrm{~mm} \mathrm{Hg}$ & 22 & $0.868(0.756-0.938)$ & $0.816(0.670-0.911)$ \\
\hline & $-20 \mathrm{~mm} \mathrm{Hg}$ & 20 & $0.811(0.654-0.913)$ & $0.817(0.663-0.916)$ \\
\hline
\end{tabular}

inspiration method is conducted through the nose, while the mouth is kept closed during a time period of approximately of 16 seconds. This results in classical "abdominal breathing". Regarding suction against resistance, in our study, pure "diaphragmatic breathing" was performed. We assume that during our suction manoeuver the diaphragm strongly squeezes the IVC explaining the pronounced IVC flow reduction. In contrast, in the case of slow inspiration, as found by Kuzo et al., the diaphragm is slowly moved downwards without any relevant resistance, which in turn increases pressure within the abdominal cavity by the organs being pushed further downwards. This, in turn, exerts indirect pressure pushing the blood of the IVC upwards resulting in an increase in non-opacified flow. This finally might very well explain the observed differences and in part the contradictory results.

Moreover, our explanation for reduced IVC flow during rapid inspiration is also confirmed by older studies. During strong and rapid inspiration, compression of the inferior vena cava by the diaphragm was described in prior physiological studies [12-14]. These results seem to confirm that IVC blood flow may well be reduced under very strong and rapid inspiration schemes.

A further reason why our data might contradict previous radiological studies is the partly confusing or unclear definition of individual breathing manoeuvers in the various publications, especially in CT studies. One example is the study by Mortimer et al. [5]. They defined inspiration and expiration as follows: in their CT investigation, patients were asked to perform an inspiration and an expiration breath with the CT investigation performed during the succeeding breath hold/apnoea phase. This is certainly not the classical type of inspiration, but a static investigation with no dynamics, either with a well-filled chest (referred to as inspiration) or a depleted chest (known as expiration). 
Besides the difficulty in distinguishing the various breathing modes, a further problem in most radiological studies performed so far was the lack of analysing repeatability and reproducibility. Kuzo [11] also did not compare repeated measurements on MRI. In our study, every breathing position measurement was repeated three times and the results were compared intraindividually. The fact that high ICC scores were registered confirms the high quality and repeatability of the standardised breathing manoeuvers.

The following limitations of the present study are worthy of note. The data were acquired from healthy volunteers using a physiological MRI investigation and, thus, cannot be simply transferred to future CT settings. The study will, therefore, have to be repeated during $\mathrm{CT}$ image acquisition, using a similar experimental setup. Apart from this, all of our volunteers were healthy and mostly young persons of normal weight. We do not know whether the data can be automatically transferred to diseased, physically impaired, or obese persons. We also do not know if patients under clinical conditions suffering strong dyspnoea are able to perform the suck against resistance manoeuver. Last but not least, the phasecontrast technique used is a non-contrast method, thus, not allowing extrapolation of the data automatically to contrast studies. All of these open questions should be addressed in future CT and MR studies.

Conclusion: Suction against resistance significantly increases blood flow in the SVC and simultaneously reduces IVC flow. Other breathing methods showed no significant influence. This distinct drop of the IVC/SVC flow ratio might theoretically promise increased contrast enhancement in pulmonary CT angiography.

Acknowledgements The scientific guarantor of this publication is Andreas Gutzeit (MD). The authors of this manuscript declare no relationships with any companies, whose products or services may be related to the subject matter of the article. The authors state that this work has not received any funding.

Institutional Review Board approval was obtained. Written informed consent was obtained from all subjects in this study. Methodology: prospective, experimental, performed at one institution.

We thank Professor Georg Bongartz from the Radiology Department of the University Hospital in Basel in Switzerland for his valuable input during the ECR 2012 lecture about influencing factors regarding contrast enhancement by pulmonary $\mathrm{CT}$. This finally served as the impetus to perform this study.

Furthermore, we would like to thank the biostatistician Nicole Graf for her continuous support in the design of the study, data evaluation, and statistical testing (www.biostatistics.ch).

\section{References}

1. Wittram C, Maher MM, Yoo AJ, Kalra MK, Shepard JA, McLoud TC (2004) CT angiography of pulmonary embolism: diagnostic criteria and causes of misdiagnosis. Radiographics 24:1219-1238

2. Stein PD, Kayali F, Hull RD (2007) Spiral computed tomography for the diagnosis of acute pulmonary embolism. Thromb Haemost 98: $713-720$

3. Rathbun SW, Raskob GE, Whitsett TL (2000) Sensitivity and specificity of helical computed tomography in the diagnosis of pulmonary embolism: a systematic review. Ann Intern Med 132:227-232

4. Gosselin MV, Rassner UA, Thieszen SL, Phillips J, Oki A (2004) Contrast dynamics during CT pulmonary angiogram: analysis of an inspiration associated artifact. J Thorac Imaging 19:1-7

5. Mortimer AM, Singh RK, Hughes J, Greenwood R, Hamilton MC (2011) Use of expiratory CT pulmonary angiography to reduce inspiration and breath-hold associated artefact: contrast dynamics and implications for scan protocol. Clin Radiol 66: $1159-1166$

6. Chen YH, Velayudhan V, Weltman DI et al (2008) Waiting to exhale: salvaging the nondiagnostic CT pulmonary angiogram by using expiratory imaging to improve contrast dynamics. Emerg Radiol 15:161-169

7. Wittram C, Yoo AJ (2007) Transient interruption of contrast on CT pulmonary angiography: proof of mechanism. J Thorac Imaging 22: $125-129$

8. Arenas-Jiménez J, Bernabé-García J, García-Espasa C (2013) Re: Use of expiratory CT pulmonary angiography to reduce inspiration and breath-hold associated artefact: contrast dynamics and implications for scan protocol. Clin Radiol 68:e98

9. Hamilton MC, Mortimer AM, Hughes J (2013) Re: Use of expiratory CT pulmonary angiography to reduce inspiration and breath-hold associated artefact: contrast dynamics and implications for scan protocol. A reply. Clin Radiol 68:e99-e100

10. Bernabé-García JM, García-Espasa C, Arenas-Jiménez J, SánchezPayá J, de la Hoz-Rosa J, Carreres-Polo JO (2012) Has "respiratory coaching" before deep inspiration an impact on the incidence of transient contrast interruption during pulmonary $\mathrm{CT}$ angiography? Insights Imaging 3:505-511

11. Kuzo RS, Pooley RA, Crook JE, Heckman MG, Gerber TC (2007) Measurement of caval blood flow with MRI during respiratory maneuvers: implications for vascular contrast opacification on pulmonary CT angiographic studies. AJR Am J Roentgenol 188:839-842

12. Wexler L, Bergel DH, Gabe IT, Makin GS, Mills CJ (1968) Velocity of blood flow in normal human venae cavae. Circ Res 23:349-359

13. Kimura BJ, Dalugdugan R, Gilcrease GW 3rd, Phan JN, Showalter BK, Wolfson $\mathrm{T}$ (2011) The effect of breathing manner on inferior vena caval diameter. Eur J Echocardiogr 12:120-123

14. Takata M, Beloucif S, Shimada M, Robotham JL (1992) Superior and inferior vena caval flows during respiration: pathogenesis of Kussmaul's sign. Am J Physiol 262:H763-H770 